\title{
Generating Distance Maps with Neighbourhood Sequences
}

\author{
Robin Strand $^{1}$, Benedek Nagy ${ }^{3, \star}$, Céline Fouard ${ }^{1}$, and Gunilla Borgefors ${ }^{2}$ \\ ${ }^{1}$ Centre for Image Analysis, Uppsala University, \\ Lägerhyddsvägen 3, SE-75237 Uppsala, Sweden \\ \{robin, celine\}@cb.uu.se \\ ${ }^{2}$ Centre for Image Analysis, Swedish University of Agricultural Sciences, \\ Lägerhyddsvägen 3, SE-75237 Uppsala, Sweden \\ gunilla@cb.uu.se \\ ${ }^{3}$ Department of Computer Science, Faculty of Informatics, University of Debrecen, \\ PO Box 12, 4010, Debrecen, Hungary and \\ Research Group on Mathematical Linguistics, Rovira i Virgili University, \\ Tarragona, Spain \\ nbenedek@inf .unideb.hu
}

\begin{abstract}
A sequential algorithm for computing the distance map using distances based on neighbourhood sequences (of any length) in the 2D square grid; and 3D cubic, face-centered cubic, and body-centered cubic grids is presented. Conditions for the algorithm to produce correct results are derived using a path-based approach. Previous sequential algorithms for this task have been based on algorithms that compute the digital Euclidean distance transform. It is shown that the latter approach is not well-suited for distances based on neighbourhood sequences.
\end{abstract}

\section{Introduction}

In [1, a sequential algorithm for computing distance transforms (DTs, where each object grid point is assigned the distance value to the closest background grid point) was introduced. The authors considered only the simple $L_{1}$ (cityblock) and $L_{\infty}$ (chessboard) metrics and they proved that a two-scan algorithm will produce a correct distance map. This is due to the fact that the distances are path-based with fixed adjacency, i.e., the distance between two points is the length of the shortest path between the points in a graph structure. For these distances, unit distance between adjacent grid points (weights) is used. The DTs obtained from $L_{1}$ and $L_{\infty}$ are very rotation-dependent. Basically, two alternative ways to decrease the rotational dependency have been introduced - weighted distances and distances based on neighbourhood sequences (n.s.distances or octagonal distances, first defined in 2]). With weighted distances (each local step is assigned a weight), the weights are allowed to have different values than one. The literature on weighted distances is rich, see for example the

\footnotetext{
^ The research is partly supported by grants OTKA F043090 and T049409.
} 
early paper [3]. Because of the fixed adjacency, the two scan algorithm applies for weighted distances on any point-lattice, 4]. With weighted distances, the rotational dependency is low also for short distances. This is not the case for n.s.-distances, where the adjacency relation is allowed to vary along the path. On the other hand, all distance values in each shortest path of length $n$ between two points consist of all integer values $1, \ldots, n$. This makes the n.s.-distances well suited for morphological operations such as dilation and erosion where the object should be divided into layers.

N.s.-distances have been considered by many authors and in most papers, the theoretical properties of n.s.-distances are examined. The theory on n.s.distances is developed in, e.g., 5,6. Distances based on neighbourhood sequences are also of value in applications and has been used for e.g. skeletonization [7] and shading of three-dimensional objects [8]. For these applications to be efficient, a fast algorithm for computing the distance transform is of great value.

The situation for n.s.-distances is a bit more complex than for weighted distances - allowing the adjacency relation to vary implies that a two-scan algorithm is not sufficient. In previous algorithms for computing the DT for n.s.distances [9, 3, 10, the scanning procedure designed for computing the Euclidean DT [9, 3, 11] were used. We will see that this approach is not appropriate for n.s.-distances.

Non-standard grids such as the face-centered cubic (fcc) grid and the bodycentered cubic (bcc) grid has gained more and more attention in the last decade. One reason is that less samples can be used with the same representation/reconstruction quality compared to the cubic grid [12:13]. For example, image acquisition techniques [12,13, image processing algorithms [14,15,16] and visualization techniques [17] have been developed for these grids.

In this paper, conditions for sequential algorithms in the square, cubic, facecentered cubic, and the body-centered cubic grids to produce correct results are derived, independently from the algorithm designed for Euclidean DT, using a path-based approach.

\section{Preliminaries}

In this paper, we will consider the square grid $\mathbb{Z}^{2}$, the cubic grid $\mathbb{Z}^{3}$, the fcc grid $\mathbb{F}$, and the bcc grid $\mathbb{B}$. When handled in parallel, $\mathbb{G}$ is used to denote all of the four grids.

Two grid points $\mathbf{x}=\left(x^{1}, x^{2}, \ldots, x^{n}\right), \mathbf{y}=\left(y^{1}, y^{2}, \ldots, y^{n}\right) \in \mathbb{Z}^{n}\left(n \in \mathbb{Z}_{+}\right)$are $\rho$-neighbours, $1 \leq \rho \leq n$, if

$$
\sum_{i=1}^{n}\left|x^{i}-y^{i}\right| \leq \rho \text { and } \max _{i \in\{1,2, \ldots, n\}}\left|x^{i}-y^{i}\right|=1 .
$$

The face-centered cubic grid $\mathbb{F}$ and the body-centered cubic grid $\mathbb{B}$ are defined as follows:

$$
\begin{aligned}
& \mathbb{F}=\left\{\left(x^{1}, x^{2}, x^{3}\right) \in \mathbb{Z}^{3}: x^{1}+x^{2}+x^{3} \equiv 0 \quad(\bmod 2)\right\} \\
& \mathbb{B}=\left\{\left(x^{1}, x^{2}, x^{3}\right) \in \mathbb{Z}^{3}: x^{1} \equiv x^{2} \equiv x^{3} \quad(\bmod 2)\right\}
\end{aligned}
$$


Two grid points $\mathbf{x}, \mathbf{y} \in \mathbb{F}$ or $\mathbb{B}$ are $\rho$-neighbours, $1 \leq \rho \leq 2$ if

$$
\sum_{i=1}^{3}\left|x^{i}-y^{i}\right| \leq 3 \text { and } \max _{i \in\{1,2,3\}}\left|x^{i}-y^{i}\right| \leq \rho .
$$

The neighbourhood relations in our four grids are visualized in Figure 1 by showing the Voronoi regions (the pixels (2D) and voxels (3D)) corresponding to some grid points.

The points $\mathbf{x}, \mathbf{y} \in \mathbb{G}$ are adjacent if $\mathbf{x}$ and $\mathbf{y}$ are $\rho$-neighbours for some $\rho$. The $\rho$-neighbours which are not $(\rho-1)$-neighbours are called strict $\rho$-neighbours. A neighbourhood sequence $B$ in $\mathbb{G}$ is a sequence $B=(b(i))_{i=1}^{\infty}$, where each $b(i)$ denotes a neighbourhood relation in $\mathbb{G}$. If $B$ is periodic, i.e., if for some fixed $l \in$ $\mathbb{Z}_{+}, b(i)=b(i+l)$ is valid for all $i \in \mathbb{Z}_{+}$, then we write $B=(b(1), b(2), \ldots, b(l))$.
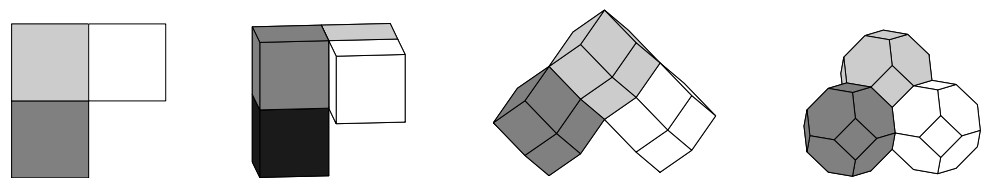

Fig. 1. The grid points corresponding to the light grey, dark grey, and black pixels/voxels are 1-, (strict) 2-, and (strict) 3-neighbours to the grid point corresponding to the white pixel/voxel, respectively. From left to right: $\mathbb{Z}^{2}, \mathbb{Z}^{3}, \mathbb{F}$, and $\mathbb{B}$.

A path, denoted $\mathcal{P}$, in a grid is a sequence $\mathbf{x}=\mathbf{p}_{0}, \mathbf{p}_{1}, \ldots, \mathbf{p}_{n}=\mathbf{y}$ of adjacent grid points. A path is a $B$-path of length $n$ if, for all $i \in\{1,2, \ldots, n\}, \mathbf{p}_{i-1}$ and $\mathbf{p}_{i}$ are $b(i)$-neighbours.

Definition 1. The $B$-distance $d(\mathbf{x}, \mathbf{y} ; B)$ between the points $\mathbf{x}$ and $\mathbf{y}$ is the length of (one of) the shortest B-path(s) between the points.

Given a path of length $n$, the following notation is used: $\omega_{i}=\mathbf{p}_{i}-\mathbf{p}_{i-1}$.

A prime vector is a vector between a grid point and an adjacent grid point. Let $\Gamma=\left\{\vec{p}_{1}, \vec{p}_{2}, \ldots, \vec{p}_{m}\right\}$. The set $\left\{\mathbf{x} \in \mathbb{G}: \mathbf{x}=\sum \alpha_{i} \vec{p}_{i}\right.$ for any $\left.\alpha_{i} \in \mathbb{R}_{+}\right\}$is called the $\Gamma$-sector.

We consider finite subsets of $\mathbb{G}$ for the algorithm:

Definition 2 (Image). The finite subset $\mathcal{I}_{\mathbb{G}}$ of $\mathbb{G}$ is denoted the image domain. We call the function $f: \mathcal{I}_{\mathbb{G}} \longrightarrow \mathbb{N}$ an image.

Definition 3 (Foreground and background). We denote the image foreground $X$ and the background $\bar{X}$. These sets have the following properties:

1. $X \subset \mathcal{I}_{\mathbb{G}}$ and $\bar{X} \subset \mathcal{I}_{\mathbb{G}}$

2. $X \cap \bar{X}=\emptyset$

3. $X \cup \bar{X}=\mathcal{I}_{\mathbb{G}}$. 


\section{The Sequential Algorithm}

Definition 4 (Distance map). Given a neighbourhood sequence $B$, the distance map, $D M_{X}$, of $f$ is a grey level image, where the value of each point of the foreground corresponds to its shortest distance to the background, i.e.

$$
D M_{X}:\left\{\begin{array}{l}
\mathcal{I}_{\mathbb{G}} \longrightarrow \mathbb{N} \\
\mathbf{x} \longmapsto d(\mathbf{x}, \bar{X} ; B)=\inf _{\mathbf{y} \in \bar{X}} d(\mathbf{x}, \mathbf{y} ; B)
\end{array}\right.
$$

Definition 5 (Scanning mask). A scanning mask $\mathcal{M}$ is the set of vectors from the origin to some grid points adjacent to $\mathbf{0}$.

Definition 6 (Scanning order). A scanning order (so) is an ordering of the $M=\operatorname{card}\left(\mathcal{I}_{\mathbb{G}}\right)$ distinct points in $\mathcal{I}_{\mathbb{G}}$, denoted $\mathbf{x}_{1}, \mathbf{x}_{2}, \ldots, \mathbf{x}_{M}$.

For a scanning mask to propagate distances correctly, it is important that, in each step of the propagation, the values at the points in $\mathcal{I}_{\mathbb{G}}$ to which the mask propagate distances will propagate distances later in the scan. This is guaranteed if each point that can be reached by the scanning mask either has not been visited or is outside the image.

Definition 7 (Mask supporting a scanning order). Let $\mathbf{x}_{1}, \mathbf{x}_{2}, \ldots, \mathbf{x}_{M}$ be a scanning order and $\mathcal{M}_{l}$ a scanning mask. The scanning mask $\mathcal{M}_{l}$ supports the scanning order if

$$
\forall \mathbf{x}_{i}, \forall \vec{v}_{j} \in \mathcal{M}_{l},\left(\left(\exists i^{\prime}>i: \mathbf{x}_{i^{\prime}}=\mathbf{x}_{i}+\vec{v}_{j}\right) \text { or }\left(\mathbf{x}_{i}+\vec{v}_{j} \notin \mathcal{I}_{\mathbb{G}}\right)\right) .
$$

Remark 1. If $\vec{v} \in \mathcal{M}_{k}$ for some $k$, then $-\vec{v} \notin \mathcal{M}_{k}$. If both $\vec{v},-\vec{v} \in \mathcal{M}_{k}$, then by Definition [7, there is an $i^{\prime}$ such that $\mathbf{x}_{i^{\prime}}=\left(\mathbf{x}_{i}+\vec{v}\right)+(-\vec{v})=\mathbf{x}_{i}$. This is not possible since each grid point occurs only once in $\mathcal{I}_{\mathbb{G}}$.

Algorithm 1. Initially, $f(\mathbf{x})=\infty$ if $\mathbf{x} \in X$ and $f(\mathbf{x})=0$ if $\mathbf{x} \in \bar{X}$. The image domain $\mathcal{I}_{\mathbb{G}}$ is scanned $L$ times using scanning orders such that the scanning masks $\mathcal{M}_{i}, 1 \leq i \leq L$ support the scanning orders so $_{i}$.

for $i=1: L$

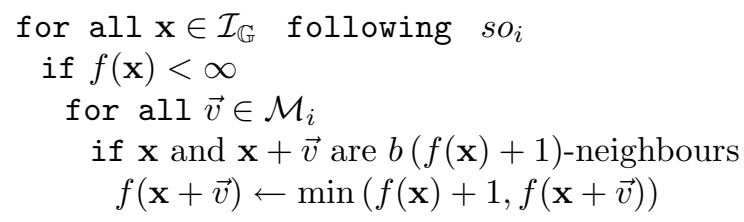

Example 1. Consider the image in $\mathbb{Z}^{2}$ shown in Figure 2(a) (the grid points are visualized by their pixels). The masks $\mathcal{M}_{1}$ and $\mathcal{M}_{2}$ are such that they propagate distances in the directions shown in Figure 2(b) by dashed and solid lines, respectively. The correct distance maps for $B=(1)$ (city block), $B=(2)$ (chessboard), and $B=(1,2)$ are shown in Figure 2(c), Figure 2(d), and Figure 2(e), respectively. For $B=(1)$ and $B=(2)$, a two-scan algorithm is sufficient to propagate the distance between the two pixels in grey. Two scans are, however, not enough for $B=(1,2)$. 


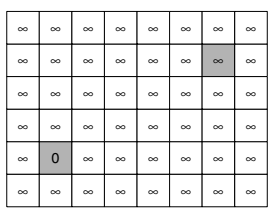

(a)

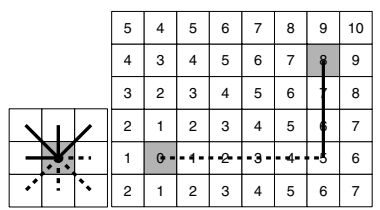

(b)

\begin{tabular}{|l|l|l|l|l|l|l|l|}
\hline 4 & 4 & 4 & 4 & 4 & 4 & 5 & 6 \\
\hline 3 & 3 & 3 & 3 & 3 & 4 & 5 & 6 \\
\hline 2 & 2 & 2 & 2 & 3 & 7 & 5 & 6 \\
\hline 1 & 1 & 1 & 2 & .6 & 4 & 5 & 6 \\
\hline 1 & 0 & -4 & -2 & 3 & 4 & 5 & 6 \\
\hline 1 & 1 & 1 & 2 & 3 & 4 & 5 & 6 \\
\hline
\end{tabular}

(d)

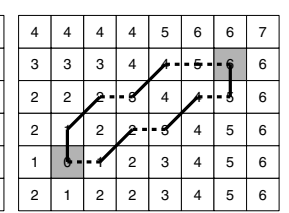

(e)

Fig. 2. Distance maps of (a) for $B=(1), B=(2)$, and $B=(1,2)$ are shown in (c), (d), and (e), respectively. Examples of shortest $B$-paths are shown in (c)-(e) over the distance maps. Directions supported by the masks $\mathcal{M}_{1}$ and $\mathcal{M}_{2}$ are shown as dashed and solid lines, respectively. The directions supported by the masks are shown in (b).

The distance that are propagated depends on previous propagations. Thus, if local steps from $\mathcal{M}_{2}$ are needed before local steps from $\mathcal{M}_{1}$ (as in Figure 2(e)), then two scans are not enough.

Since Algorithm 1 only propagates distances from mask $i$ in scan $i$, there must be a shortest path satisfying the condition in Proposition 1 below for each pair of grid points in $\mathcal{I}_{\mathbb{G}}$ for the propagation of distances to be sufficient.

Proposition 1. If for each neighbourhood sequence $B$ and each $\mathbf{x}, \mathbf{y} \in \mathcal{I}_{\mathbb{G}}$ there is a shortest $B$-path in $\mathcal{I}_{\mathbb{G}}$ between $\mathbf{x}$ and $\mathbf{y}$ of length $n$ and integers $T_{0}, T_{1}, \ldots$, $T_{L}$ s.t.

$$
\omega_{i} \in\left\{\begin{array}{ccc}
\mathcal{M}_{1} \text { if } & 0=T_{0}<i \leq T_{1} \\
\mathcal{M}_{2} \text { if } & T_{1}<i \leq T_{2} \\
\vdots & \\
\mathcal{M}_{L} \text { if } & T_{L-1}<i \leq T_{L}=n
\end{array}\right.
$$

then Algorithm 1 returns a distance map $D M_{X}$.

The proof of this proposition is obvious: the first scan propagates the $T_{1}$ first steps of the path, the second scan propagates the steps $T_{1}+1, \ldots, T_{2}$ and so on until the last scans propagates the steps $T_{L-1}+1, \ldots, T_{L}$.

In the next section, conditions for the masks $\mathcal{M}_{i}$ to fulfill the condition in Proposition 1 are derived.

\section{Theoretic Results}

\subsection{Conditions for a Path to Be in $\mathcal{I}_{\mathbb{G}}$}

Definition 8 (Sector-preserving image domain). The image domain is called sector-preserving if there are integers $L_{i}$ and $U_{i}$ s.t. $L_{i} \leq 0<U_{i}$ and

$$
\begin{array}{r}
\mathcal{I}_{\mathbb{Z}^{2}}=\left\{\left(x^{1}, x^{2}\right) \in \mathbb{Z}^{2} \mid L_{i} \leq x^{i} \leq U_{i}\right\} \\
\mathcal{I}_{\mathbb{Z}^{3}}=\left\{\left(x^{1}, x^{2}, x^{3}\right) \in \mathbb{Z}^{3} \mid L_{i} \leq x^{i} \leq U_{i}\right\}
\end{array}
$$




$$
\begin{aligned}
& \mathcal{I}_{\mathbb{F}}=\left\{\left(x^{1}, x^{2}, x^{3}\right) \in \mathbb{F} \mid L_{1} \leq x^{1}+x^{2}+x^{3} \leq U_{1}, L_{2} \leq x^{1}+x^{2}-x^{3} \leq U_{2},\right. \\
& \left.L_{3} \leq x^{1}-x^{2}-x^{3} \leq U_{3} \text {, and } L_{4} \leq x^{1}-x^{2}+x^{3} \leq U_{4}\right\} \\
& \mathcal{I}_{\mathbb{B}}=\left\{\left(x^{1}, x^{2}, x^{3}\right) \in \mathbb{B} \mid L_{1} \leq x^{1}+x^{2} \leq U_{1}, L_{2} \leq x^{2}+x^{3} \leq U_{2}\right. \text {, and } \\
& \left.L_{3} \leq x^{1}+x^{3} \leq U_{3}\right\} \text {. }
\end{aligned}
$$

Let

$$
\begin{aligned}
\Gamma_{\mathbb{Z}^{2}}^{1} & =\{(1,0),(0,1),(1,1)\} \\
\Gamma_{\mathbb{Z}^{3}}^{1} & =\{(1,0,0),(0,1,0),(0,0,1),(1,1,0),(1,0,1),(0,1,1),(1,1,1)\} \\
\Gamma_{\mathbb{F}}^{1} & =\{(1,1,0),(1,0,1),(0,1,1)\} \\
\Gamma_{\mathbb{F}}^{2} & =\{(1,1,0),(1,0,1),(1,0,-1),(2,0,0)\} \\
\Gamma_{\mathbb{B}}^{1} & =\{(1,1,1),(1,-1,1),(1,1,-1),(2,0,0)\} .
\end{aligned}
$$

Definition 9 (Path with $\Gamma_{\mathbb{G}}$-sector steps). A path that contains only steps from $\Gamma_{\mathbb{G}}$ is called a path with $\Gamma_{\mathbb{G}}$-sector steps.

We will see that for any fixed $\xi$ and any grid point $\mathbf{x} \in \mathcal{D}_{\mathbb{G}}^{\xi}$ defined below, the distance (i.e. the shortest path) between $\mathbf{0}$ and $\mathbf{x}$ is defined by a path with $\Gamma_{\mathbb{G}}^{\xi}$-sector steps.

$$
\begin{aligned}
\mathcal{D}_{\mathbb{Z}^{2}}^{1} & =\left\{\mathrm{x} \in \mathbb{Z}^{2}: x^{i} \geq 0\right\} \\
\mathcal{D}_{\mathbb{Z}^{3}}^{1} & =\left\{\mathrm{x} \in \mathbb{Z}^{3}: x^{i} \geq 0\right\} \\
\mathcal{D}_{\mathbb{F}}^{1} & =\left\{\mathrm{x} \in \mathbb{F}: x^{1} \geq x^{2} \geq x^{3} \geq 0 \text { and } x^{1} \leq x^{2}+x^{3}\right\} \\
\mathcal{D}_{\mathbb{F}}^{2} & =\left\{\mathrm{x} \in \mathbb{F}: x^{1} \geq x^{2} \geq x^{3} \geq 0 \text { and } x^{1}>x^{2}+x^{3}\right\} \\
\mathcal{D}_{\mathbb{B}}^{1} & =\left\{\mathrm{x} \in \mathbb{B}: x^{1} \geq x^{2} \geq x^{3} \geq 0\right\}
\end{aligned}
$$

We also will use the following notation: for any $\mathbf{y} \in \mathbb{G}, \mathcal{D}_{\mathbb{G}}(\mathbf{y})=\left\{\mathbf{y}+\mathbf{x}: \mathbf{x} \in \mathcal{D}_{\mathbb{G}}\right\}$. The following proposition follows directly from Theorem 3.5 in $[18]\left(\mathbb{Z}^{2}\right.$ and $\left.\mathbb{Z}^{3}\right)$ and from the proofs of Theorem 2 and 5 in [15] for $\mathbb{F}$ and $\mathbb{B}$ respectively.

Proposition 2. Let the neighbourhood sequence $B$ and the $\xi$ be given. For any point $\mathbf{y} \in \mathcal{D}_{\mathbb{G}}^{\xi}(\mathbf{x})$ such that $d(\mathbf{x}, \mathbf{y} ; B)=n$, there is a shortest B-path between $\mathbf{x}$ and $\mathbf{y}$ with $\Gamma_{\mathbb{G}}^{\xi}$-sector steps.

For the square/cubic grid it is obvious that we need vectors having a value 1 and one/two zero(s) to connect the points by only 1-steps. If the neighbourhood sequence contains values 2 and there are coordinate differences in at least 2 coordinates, then vectors changing 2 values simultaneously can be used in a shortest path. In the cubic grid $(1,1,1)$ is used if the neighbourhood sequence contains element 3 and there are differences in all the 3 coordinates.

In the fcc grid if $x^{1} \leq x^{2}+x^{3}$ then vectors $(1,1,0),(1,0,1),(0,1,1)$ can produce a shortest path independently of the used neighbourhood sequence (and in case $B=(1)$ all shortest paths built by them).

In case of $x^{1}>x^{2}+x^{3}$ then the steps $(0,1,1)$ are not needed, but we need (additional) steps to go in direction $\left(x^{1}, 0,0\right)$. If there are values 2 in the neighbourhood sequence, then vector $(2,0,0)$ is used in a shortest path. Having not 
(enough) values 2 in the neighbourhood sequence a step by $(2,0,0)$ can be substituted by a step $(1,0,1)$ and a step $(1,0,-1)$.

In the bcc grid one can construct a shortest path by vectors $(1,1,1)$ and $(1,1,-1)$ if $x^{1}=x^{2} \geq x^{3} \geq 0$. If $x^{1}>x^{2}$ then $\operatorname{step}(\mathrm{s})$ by vector $(2,0,0)$ can also be used having values 2 in the neighbourhood sequence. Without (enough) values 2 in the neighbourhood sequence steps by vectors $(1,1,-1)$ and $(1,-1,1)$ are also used in a shortest path.

Definition 10 (Image border point). All grid points $\mathbf{x} \in \mathcal{I}_{\mathbb{G}}$ such that there is a prime vector $\vec{v}$ such that $\mathbf{x}+\vec{v} \notin \mathcal{I}_{\mathbb{G}}$ are called image border points.

Lemma 1. Let $\mathbf{y} \in \bar{X}$ and $\mathbf{x} \in X$ be such that $\mathbf{x} \in \mathcal{D}_{\mathbb{G}}^{\xi}(\mathbf{y})$ for some $\xi$ and there is a shortest $B$-path $\mathcal{P}$ between $\mathbf{y}$ and $\mathbf{x}$ defining $d(\mathbf{x}, \bar{X} ; B)$. If either

(A) all image border points are in the background or

(B) the image domain is sector-preserving.

then $\mathcal{P}$ is in $\mathcal{I}_{\mathbb{G}}$.

Proof. When condition (A) is fulfilled: For some $\mathbf{y} \in \bar{X}$, let $\mathbf{x}=\mathbf{p}_{0}, \mathbf{p}_{1}, \ldots, \mathbf{p}_{n}=$ $\mathbf{y}$ be the $B$-path defining $d(\mathbf{x}, \bar{X} ; B)=n$. Let $\mathbf{y}=\mathbf{q}_{0}, \mathbf{q}_{1}, \ldots, \mathbf{q}_{n}=\mathbf{x}$ be the $B$ path defining $d(\mathbf{y}, \mathbf{x} ; B)=n$ and assume that $\mathbf{q}_{i} \notin \mathcal{I}_{\mathbb{G}}$ for some $i, 0<i<n$. Let $\mathcal{S}_{\mathbb{G}, B}(\mathbf{x}, k)=\{\mathbf{y}: d(\mathbf{x}, \mathbf{y} ; B)=k\}$. Now, $\mathbf{q}_{i} \in \mathcal{S}_{\mathbb{G}, B}(\mathbf{x}, k)$ for some $k \leq n$. Therefore, by Definition 10, there is a background grid point $\mathbf{z} \in \mathcal{S}_{\mathbb{G}, B}(\mathbf{x}, k-1)$ such that $\mathbf{q}_{i}$ and $\mathbf{z}$ are adjacent. We thus have $n=d(\mathbf{x}, \bar{X} ; B) \leq d(\mathbf{x}, \mathbf{z} ; B)=k-1<n$, which is a contradiction.

When condition (B) is fulfilled: Let $\mathbf{y}=\mathbf{p}_{0}, \ldots, \mathbf{p}_{n}=\mathbf{x}$ be a shortest path with $\Gamma_{\mathbb{G}}^{\xi}$-sector steps between $\mathbf{y}$ and $\mathbf{x}$. Assume that there is an $i(0 \leq i \leq n)$ such that $\mathbf{p}_{i} \notin \mathcal{I}_{\mathbb{G}}$. Since $\mathbf{y} \in \mathcal{I}_{\mathbb{G}}$, this implies

- $\left[\mathbb{Z}^{2}, \mathbb{Z}^{3}\right] p_{i}^{j}>U_{j}$ for some $j$.

$-\left[\mathbb{F}, \mathcal{D}^{1}\right] p_{i}^{1}+p_{i}^{2}+p_{i}^{3}>U_{1}, p_{i}^{1}+p_{i}^{2}-p_{i}^{3}>U_{2}, p_{i}^{1}-p_{i}^{2}-p_{i}^{3}<L_{3}$, or $p_{i}^{1}-p_{i}^{2}+p_{i}^{3}>U_{4}$.

$-\left[\mathbb{F}, \mathcal{D}^{2}\right] p_{i}^{1}+p_{i}^{2}+p_{i}^{3}>U_{1}, p_{i}^{1}+p_{i}^{2}-p_{i}^{3}>U_{2}, p_{i}^{1}-p_{i}^{2}-p_{i}^{3}>U_{3}$, or $p_{i}^{1}-p_{i}^{2}+p_{i}^{3}>U_{4}$.

$-[\mathbb{B}] p_{i}^{1}+p_{i}^{2}>U_{1}, p_{i}^{2}+p_{i}^{3}>U_{2}$, or $p_{i}^{1}+p_{i}^{3}>U_{3}$.

Now, for all local steps in $\Gamma_{\mathbb{G}}^{\xi}$, these inequalities are valid also for $\mathbf{p}_{i+1}$. Thus $\mathbf{p}_{j} \notin \mathcal{I}_{\mathbb{G}}$ for all $j \geq i$, which contradicts $\mathbf{x} \in \mathcal{I}_{\mathbb{G}}$.

\subsection{Rules to Swap Steps in a Path}

Lemma 2. Let the neighbourhood sequence $B$ and the image $\mathcal{I}_{\mathbb{G}}$ be such that either the image domain is sector-preserving or all image border points are in the background. Let $\mathcal{I}_{\mathbb{G}}$ be such that $\mathbf{y} \in \bar{X}$ and let $\mathbf{x} \in X \cap \mathcal{D}_{\mathbb{G}}^{\xi}(\mathbf{y})$ for some $\xi$ be such that the $B$-path $\mathcal{P}$ with $\Gamma_{\mathbb{G}}^{\xi}$-sector steps of length $n$ between $\mathbf{x}$ and $\mathbf{y}$ is a 
shortest path defining $d(\mathbf{x}, \bar{X} ; B)$. For any $k(1 \leq k \leq n)$, let $\rho_{k}$ be such that $\omega_{k}$ corresponds to a strict $\rho_{k}$-neighbour. Let also $i, j$ be two fixed integers such that $1 \leq i, j \leq n$.

Then for any $\omega_{i}^{\prime}$ and $\omega_{j}^{\prime}$ such that

(A1) $\omega_{i}^{\prime}, \omega_{j}^{\prime} \in \Gamma_{\mathbb{G}}^{\xi}$

(A2) $\omega_{i}^{\prime}$ and $\omega_{j}^{\prime}$ correspond to strict $\rho_{i}$ - and $\rho_{j}$-neighbours respectively,

(A3) $\omega_{i}+\omega_{j}=\omega_{i}^{\prime}+\omega_{j}^{\prime}$

there is a shortest $B$-path $\mathcal{P}^{\prime}$ in $\mathcal{I}_{\mathbb{G}}$ of length $n$ between $\mathbf{0}$ and $\mathbf{x}$ such that $\omega_{k}^{\prime}=\omega_{k}$ if $k \neq i, j$ and $\omega_{i}^{\prime}=\omega_{j}$ and $\omega_{j}^{\prime}=\omega_{i}$.

Proof. Let $\mathcal{P}$ be the path $\mathbf{x}=\mathbf{p}_{0}, \mathbf{p}_{1}, \ldots, \mathbf{p}_{i-1}, \mathbf{p}_{i}, \ldots, \mathbf{p}_{j-1}, \mathbf{p}_{j}, \ldots, \mathbf{p}_{n}=\mathbf{y}$ and let $\omega_{i}^{\prime}$ and $\omega_{j}^{\prime}$ satisfy $(\mathrm{A} 1)-(\mathrm{A} 3)$. We have

$$
\begin{array}{cl}
\omega_{i}=\mathbf{p}_{i}-\mathbf{p}_{i-1}, & \omega_{i}^{\prime}=\mathbf{p}_{i}^{\prime}-\mathbf{p}_{i-1} \\
\omega_{j}=\mathbf{p}_{j}-\mathbf{p}_{j-1}, & \omega_{j}^{\prime}=\mathbf{p}_{j}-\mathbf{p}_{j-1}^{\prime} \\
\omega_{i}+\omega_{j}=\omega_{i}^{\prime}+\omega_{j}^{\prime} & (\text { by }(\text { A3) }) .
\end{array}
$$

It follows from Equations (3)-(5) that $\mathbf{p}_{i}-\mathbf{p}_{i}^{\prime}=\mathbf{p}_{j-1}-\mathbf{p}_{j-1}^{\prime}$, i.e., that $\mathbf{p}_{i}^{\prime}=\mathbf{p}_{i}-\vec{v}$ and $\mathbf{p}_{j-1}^{\prime}=\mathbf{p}_{j-1}-\vec{v}$ for some $\vec{v}$.

We will now see that the path $\mathcal{P}^{\prime}$, $\mathbf{x}=\mathbf{p}_{0}, \mathbf{p}_{1}, \ldots, \mathbf{p}_{i-1}, \mathbf{p}_{i}^{\prime}, \ldots, \mathbf{p}_{j-1}^{\prime}, \mathbf{p}_{j}, \ldots, \mathbf{p}_{n}=\mathbf{y}$ defined as

$$
\begin{gathered}
\mathbf{p}_{k}^{\prime}=\mathbf{p}_{k}-\vec{v} \quad \text { for any } i \leq k<j \\
\mathbf{p}_{k}^{\prime}=\mathbf{p}_{k} \text { otherwise. }
\end{gathered}
$$

is a shortest $B$-path in $\mathcal{I}_{\mathbb{G}}$ satisfying the Lemma.

By $(\mathrm{A} 1), \mathcal{P}^{\prime}$ is a path with $\Gamma_{\mathbb{G}}^{\xi}$-sector steps. Thus, by Lemma 1 , $\mathcal{P}^{\prime}$ is in the image $\mathcal{I}_{\mathbb{G}}$.

By the definition of $\mathcal{P}^{\prime}$, it is a shortest path (since it is of the same length as $\mathcal{P})$. Moreover, $\omega_{k}=\mathbf{p}_{k}-\mathbf{p}_{k-1}=\mathbf{p}_{k}^{\prime}-\mathbf{p}_{k-1}^{\prime}=\omega_{k}^{\prime}$ for any $i<k<j$, so $\mathbf{p}_{k}$ and $\mathbf{p}_{k-1}$ are strict $\rho$-neighbours if and only if $\mathbf{p}_{k}^{\prime}$ and $\mathbf{p}_{k-1}^{\prime}$ are. The cases $k=i$ and $k=j$ follow from (A2) and when $k<i$ or $j<k, \mathbf{p}_{k}^{\prime}=\mathbf{p}_{k}$ by definition, so $\mathcal{P}^{\prime}$ is a $B$-path.

We can now conclude that $\mathcal{P}^{\prime}$ is a shortest $B$-path in $\mathcal{I}_{\mathbb{G}}$ between $\mathbf{x}$ and $\mathbf{y}$.

Example 2. Consider $\mathbb{F}$, the neighbourhood sequence $B=(1,2,1,1,2)$ and the grid point $\mathbf{x}=(6,2,0)$. A shortest $B$-path between $\mathbf{0}$ and $\mathbf{x}$ is

$$
\mathbf{0}=(0,0,0),(1,1,0),(3,1,0),(4,1,-1),(5,1,0),(6,2,0)=\mathbf{x} .
$$

We have

$$
\omega_{1}=(1,1,0), \omega_{2}=(2,0,0), \omega_{3}=(1,0,-1), \omega_{4}=(1,0,1), \omega_{5}=(1,1,0) .
$$

By Lemma 2, the $B$-paths between $\mathbf{0}$ and $\mathbf{x}$ with the following local steps are also shortest paths.

$$
\begin{array}{ll}
\omega_{1}=(1,1,0), \quad \omega_{2}=(2,0,0), \quad \omega_{3}=(1,1,0) & \omega_{4}=(1,0,-1), \quad \omega_{5}=(1,0,1) \\
\omega_{1}=(1,0,1), & \omega_{2}=(2,0,0), \quad \omega_{3}=(1,0,-1), \quad \omega_{4}=(1,1,0), \quad \omega_{5}=(1,1,0)
\end{array}
$$


Thus, any order of the local steps corresponding to 1-neighbours results in shortest $B$-paths.

Now we define sets $\mathbf{B}_{i}^{\mathbb{G}}$. We will see that if each of these $\mathbf{B}_{i}^{\mathbb{G}}:$ s are in at least one mask (supporting the scan orders) in Algorithm 1 then Algorithm 1 propagate correct distance to any $\mathbf{x} \in \mathcal{D}_{\mathbb{G}}^{\xi}$.

$$
\begin{aligned}
& \mathbf{B}_{1}^{\mathbb{Z}^{2}}=\{(1,0), \quad(1,1)\}\left|\mathbf{B}_{1}^{\mathbb{F}}=\{(1,1,0), \quad(2,0,0)\}\right| \mathbf{B}_{1}^{\mathbb{B}}=\{(1,1,1), \quad(2,0,0)\} \\
& \mathbf{B}_{2}^{\mathbb{Z}^{2}}=\{(0,1), \quad(1,1)\} \mathbf{B}_{2}^{\mathbb{F}}=\{(1,0,1), \quad(2,0,0)\} \mathbf{B}_{2}^{\mathbb{B}}=\{(1,-1,1), \quad(2,0,0)\} \\
& \mathbf{B}_{3}^{\mathbb{F}}=\{(0,1,1), \quad(2,0,0)\} \mathbf{B}_{3}^{\mathbb{B}}=\{(1,1,-1),(2,0,0)\} \\
& \left|\mathbf{B}_{3}^{\mathbb{F}}=\{(1,0,-1),(2,0,0)\}\right| \\
& \mathbf{B}_{1}^{\mathbb{Z}^{3}}=\{(1,0,0),(1,1,0),(1,1,1)\} \mid \mathbf{B}_{4}^{\mathbb{Z}^{3}}=\{(0,1,0),(0,1,1),(1,1,1)\} \\
& \mathbf{B}_{2}^{\mathbb{Z}^{3}}=\{(1,0,0),(1,0,1),(1,1,1)\} \mathbf{B}_{5}^{\mathbb{Z}^{3}}=\{(0,0,1),(0,1,1),(1,1,1)\} \\
& \mathbf{B}_{3}^{\mathbb{Z}^{3}}=\{(0,1,0),(1,1,0),(1,1,1)\} \mid \mathbf{B}_{6}^{\mathbb{Z}^{3}}=\{(0,0,1),(1,0,1),(1,1,1)\}
\end{aligned}
$$

\subsection{Minimal Configuration of Scanning Masks}

Theorem 1. Let the set $\alpha_{i}, 1 \leq i \leq L$ such that $\alpha_{i} \neq \alpha_{k}$ if $i \neq k$ and $\alpha_{i} \in$ $\{1,2, \ldots, L\}$ for all $i$ be given. For any neighbourhood sequence $B$, any image $\mathcal{I}_{\mathbb{G}}$ such that all image border points are in the background, and any points $\mathbf{y} \in \bar{X}$ and $\mathbf{x} \in X \cap \mathcal{D}_{\mathbb{G}}^{\xi}(\mathbf{y})$ such that $d(\mathbf{x}, \mathbf{y})=d(\mathbf{x}, \bar{X} ; B)$ there is a shortest path between $\mathbf{x}$ and $\mathbf{y}$ such that

$$
\begin{gathered}
\omega_{j} \in \mathbf{B}_{\alpha_{1}}^{\mathbb{G}} \text { if } 0=k_{0}<j \leq k_{1} \\
\omega_{j} \in \mathbf{B}_{\alpha_{2}}^{\mathbb{G}} \text { if } k_{1}<j \leq k_{2} \\
\vdots \\
\omega_{j} \in \mathbf{B}_{\alpha_{L}}^{\mathbb{G}} \text { if } k_{L-1}<j \leq k_{L}
\end{gathered}
$$

for some $k_{i}: s$.

Proof. The theorem follows directly from Lemma 2 for $\mathbb{Z}^{2}, \mathbb{F}$, and $\mathbb{B}$. Since the only 2-neighbour $\left((1,1),(2,0,0)\right.$, and $(2,0,0)$, respectively) is in all the $\mathbf{B}_{i}^{\mathbb{G}}: \mathrm{s}$, it is enough to order the 1-neighbours such that (7)-(9) are fulfilled. Compare with Example 2,

For $\mathbb{Z}^{3}$, things are a bit more complicated. We argue by contradiction. Let $\mathcal{P}$ be any shortest $B$-path (of length $n$ ) with $\Gamma_{\mathbb{G}}$-sector steps between $\mathbf{x}$ and $\mathbf{y}$. Construct the new path $\mathcal{P}^{\prime}$ as follows:

There is obviously a maximal value of $k_{1}$ such that $\omega_{j} \in \mathbf{B}_{\alpha_{1}}^{\mathbb{Z}^{3}}$ for all $0<j \leq k_{1}$. Lemma 2 is used to find the $\omega_{j}$ :s. In the same way, maximal values of $k_{2}, \ldots, k_{6}$ and the $\omega_{j}:$ s for $j \leq k_{6}$ are found.

Assume that $k_{6} \neq n$ (i.e. that $k_{6}<n$ ). Since $(1,1,1)$ is in all $\mathbf{B}_{i}^{\mathbb{Z}^{3}}: \mathrm{s}, \omega_{k_{6}+1}$ corresponds either to a 1-neighbour or a 2-neighbour. 
Case $i \omega_{k_{6}+1}$ corresponds to a 1-neighbour.

We consider $\omega_{k_{6}+1}=(1,0,0)$ - the proofs for $(0,1,0)$ and $(0,0,1)$ are similar.

Let $a$ and $b$ be the values such that $\omega_{j} \in \mathbf{B}_{1}^{\mathbb{Z}^{3}}$ if $k_{a-1}<j \leq k_{a}$ and $\omega_{j} \in \mathbf{B}_{2}^{\mathbb{Z}^{3}}$ if $k_{b-1}<j \leq k_{b}$. We assume $b>a$ (the proof for $a>b$ is similar).

Since $(1,1,1)$ is in all $\mathbf{B}_{i}^{\mathbb{Z}^{3}}: \mathrm{s}$, neither $\omega_{k_{a}+1}$ nor $\omega_{k_{b}+1}$ corresponds to 3 neighbours.

By Lemma 2, neither $\omega_{k_{a}+1}$ nor $\omega_{k_{b}+1}$ corresponds to 1-neighbours: If, say $\omega_{k_{a}+1}$ corresponds to a 1-neighbour, then we could use Lemma 2 to swap $\omega_{k_{a}+1}$ and $\omega_{k_{6}+1}$ contradicting that $k_{a}$ is maximal.

Thus both $\omega_{k_{a}+1}$ and $\omega_{k_{b}+1}$ correspond to 2-neighbours. It follows that $\omega_{j} \neq$ $(1,1,0)$ for $j>k_{a}$ and $\omega_{j} \neq(1,0,1)$ for $j>k_{b}$. (Otherwise we could use Lemma 2 to swap any such occurence of $(1,1,0)$ or $(1,0,1)$ with $\omega_{k_{a}+1}$ or $\omega_{k_{b}+1}$ contradicting that $k_{a}$ and $k_{b}$ are maximal.)

Thus, $\omega_{k_{b}+1}=(0,1,1)$. But then we could use Lemma 2 to set $\omega_{k_{b}+1}$ to $(1,0,1)$ and $\omega_{k_{6}+1}$ to $(0,1,0)$ contradicting that $k_{b}$ is maximal.

Case ii $\omega_{k_{6}+1}$ corresponds to a 2-neighbour.

We consider $\omega_{k_{6}+1}=(1,1,0)$ (the proofs for $(1,0,1)$ and $(0,1,1)$ are similar).

Let now $a$ and $b$ be the values such that $\omega_{j} \in \mathbf{B}_{1}^{\mathbb{Z}^{3}}$ if $k_{a-1}<j \leq k_{a}$ and $\omega_{j} \in \mathbf{B}_{3}^{\mathbb{Z}^{3}}$ if $k_{b-1}<j \leq k_{b}$. We assume $b>a-$ the proof for $a>b$ is similar.

Since $(1,1,1)$ is in all $\mathbf{B}_{i}^{\mathbb{Z}^{3}}: \mathrm{s}$, neither $\omega_{k_{a}+1}$ nor $\omega_{k_{b}+1}$ corresponds to 3 neighbours.

By Lemma 2, neither $\omega_{k_{a}+1}$ nor $\omega_{k_{b}+1}$ corresponds to 2-neighbours: If, say $\omega_{k_{a}+1}$ corresponds to a 2 -neighbour, then we could use Lemma 2 to swap $\omega_{k_{a}+1}$ and $\omega_{k_{6}+1}$ contradicting that $k_{a}$ is maximal.

Thus both $\omega_{k_{a}+1}$ and $\omega_{k_{b}+1}$ correspond to 1-neighbours. It follows that $\omega_{j} \neq$ $(1,0,0)$ for $j>k_{a}$ and $\omega_{j} \neq(0,1,0)$ for $j>k_{b}$. (Otherwise we could use Lemma 2 to swap any such occurence of $(1,0,0)$ or $(0,1,0)$ with $\omega_{k_{a}+1}$ or $\omega_{k_{b}+1}$ contradicting that $k_{a}$ and $k_{b}$ are maximal.)

Thus, $\omega_{k_{b}+1}=(0,0,1)$. But then we could use Lemma 2 to set $\omega_{k_{b}+1}$ to $(0,1,0)$ and $\omega_{k_{6}+1}$ to $(1,0,1)$ contradicting that $k_{b}$ is maximal.

Now we can conclude that, since all possible $\omega_{k_{6}+1}$ :s lead to contradictions, $k_{6}=n$ and the proof is finished.

We can now conclude that since the order of the $\mathbf{B}_{i}^{\mathbb{G}}: \mathrm{s}$ is arbitrary, Algorithm 1 will propagate the correct distance value from $\mathbf{y} \in \bar{X}$ to each point in $\mathcal{D}_{\mathbb{G}}(\mathbf{y})$ if each $\mathbf{B}_{i}^{\mathbb{G}}: \mathrm{s}$ is included in at least one mask in the algorithm. By symmetry, it follows that if all configurations symmetric to the $\mathbf{B}_{i}^{\mathbb{G}}: \mathrm{s}$ are included in at least one mask in the algorithm, distance values will be propagated from $\mathbf{y}$ to any object grid point in $\mathcal{I}_{\mathbb{G}}$. We get the following condition:

Condition 1. If each configuration symmetric to the configurations shown in Figure 1 is included in at least one mask supporting the scan orders used in Algorithm 1, then Algorithm 1] will produce correct distance maps.

Sets of masks in the different grids fulfilling Condition 1 are shown in Figure 3. 


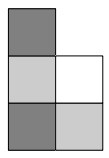

(a)

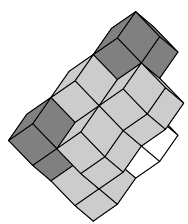

(h)

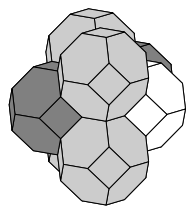

(1)

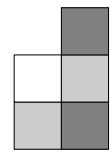

(b)

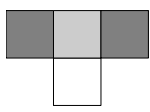

(c)

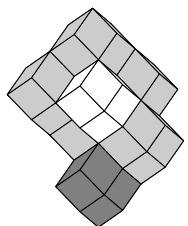

(i)

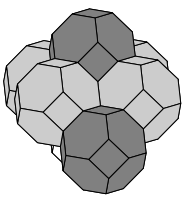

(m)

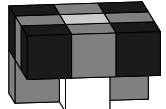

(d)

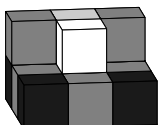

(e)

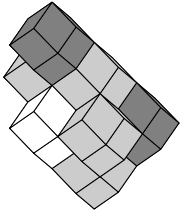

(j)

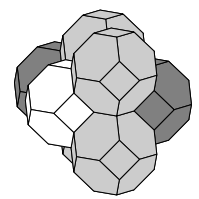

(n)

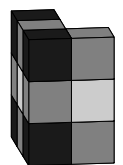

(f)

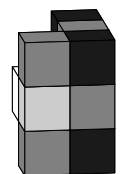

(g)

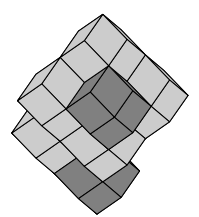

(k)

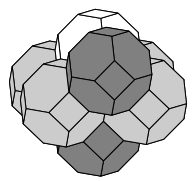

(o)

Fig. 3. The masks above (colour-coded as in Figure 1) can be used to get correct results from Algorithm 1 (a)-(c) $\mathbb{Z}^{2},(\mathrm{~d})-(\mathrm{g}) \mathbb{Z}^{3},(\mathrm{~h})-(\mathrm{k}) \mathbb{F}$, and (l)-(o) $\mathbb{B}$. Note that, for $\mathbb{Z}^{3}$, $\mathbb{F}$, and $\mathbb{B}$, any mask can be obtained by rotating any other mask in the same grid.

\section{Discussion and Conclusion}

The unfolded cube graph was introduced in 11 to guarantee that local distances are allowed to propagate in all possible directions. It was designed for Euclidean DTs and shows the directions supported by a mask. The unfolded cube graphs for a set of masks must fill the whole cube (direction space) to produce correct Euclidean DTs. It is easy to produce a set of masks such that the unfolded cube graph is covered but which does not produce correct distance maps for n.s.distances as shown in Figure 4. We have shown that distance propagation from all directions is not sufficient for algorithms for n.s.-distances. The condition derived in this paper is: if each configuration symmetric to the configuration in Figure 1 is contained in at least one mask, then the algorithm produces correct DT:s. We can notice that these configurations contain one and only one vector of each neighbourhood kind.
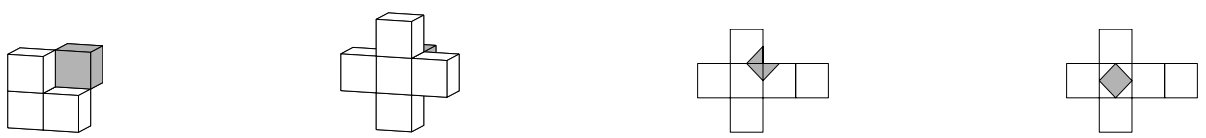

Fig. 4. Using these masks and the $7+5$ symmetric masks for $\mathbb{Z}^{3}$ in Algorithm 1 will result in insufficient propagation of distances, e.g., for neighbourhood sequences with $B=(1,3)$ 
In [15], scanning masks to compute the Euclidean DT on the fcc and bcc grids were derived using the unfolded cube graph. Surprisingly, four scans are not sufficient for the bcc grid - five masks, i.e. scans, are needed to fill the direction space using the unfolded cube graph. This implies that the number of scans needed for a sequential algorithm designed for computing the Euclidean DT might be greater than for computing n.s.-DTs.

Using the algorithms presented in this paper, applications such as skeletonization [7] and shading of three-dimensional objects [8] become faster and easier to use. Also, since sequential algorithms for the non-standard fcc and bcc grids are presented, it is easy to adjust the applications to work also for the fcc and bcc grids.

\section{References}

1. Rosenfeld, A., Pfaltz, J.L.: Sequential operations in digital picture processing. Journal of the ACM 13(4) (1966) 471-494

2. Rosenfeld, A., Pfaltz, J.L.: Distance functions in digital pictures. Pattern Recognition 1(1) (1968) 33-61

3. Borgefors, G.: Distance transformations in arbitrary dimensions. Computer Vision, Graphics, and Image Processing 27 (1984) 321-345

4. Fouard, C., Strand, R., Borgefors, G.: Weighted distance transforms generalized to modules and their computation on point lattices. Technical report, Centre for Image Analysis, Uppsala University, Uppsala, Sweden (2006) Internal report 38.

5. Yamashita, M., Honda, N.: Distance functions defined by variable neighbourhood sequences. Pattern Recognition 17(5) (1984) 509-513

6. Das, P.P., Chakrabarti, P.P., Chatterji, B.N.: Distance functions in digital geometry. Information Sciences 42 (1987) 113-136

7. Kumar, M.A., Chatterji, B.N., Mukherjee, J., Das, P.P.: Representation of 2D and 3D binary images using medial circles and spheres. International Journal of Pattern Recognition and Artificial Intelligence 10(4) (1996) 365-387

8. Mukherjee, J., Kumar, M.A., Chatterji, B.N., Das, P.P.: Discrete shading of three-dimensional objects from medial axis transform. Pattern Recognition Letters 20(14) (1999) 1533-1544

9. Danielsson, P.E.: Euclidean distance mapping. Computer Graphics and Image Processing 14 (1980) 227-248

10. Danielsson, P.E.: Minimal error octagonal metric in two and three dimensions. Internal report LiTH-ISY-1-1382, Linköping University, Linköping, Sweden (1992)

11. Ragnemalm, I.: The Euclidean distance transform in arbitrary dimensions. Pattern Recognition Letters 14(11) (1993) 883-888

12. Matej, S., Lewitt, R.M.: Efficient 3D grids for image reconstruction using spherically-symmetric volume elements. IEEE Transactions on Nuclear Science 42(4) (1995) 1361-1370

13. Garduno, E., Herman, G.T.: Optimization of basis functions for both reconstruction and visualization. Electronic Notes in Theoretical Computer Science 46 (2001) $1-17$

14. Strand, R., Borgefors, G.: Distance transforms for three-dimensional grids with non-cubic voxels. Computer Vision and Image Understanding 100(3) (2005) 294311 
15. Strand, R.: The Euclidean distance transform applied to the fcc and bcc grids. In Marques, J.S., de la Blanca, N.P., Pina, P., eds.: IbPRIA 2005, Estoril, Portugal, June 7-9, 2005, Proceedings, Volume 3522 of LNCS., Springer (2005) 243-250

16. Carvalho, B.M., Garduno, E., Herman, G.T.: Multiseeded fuzzy segmentation on the face centered cubic grid. In Ltd., S.V., ed.: Proceedings ICAPR 2001, Pattern Analysis and Applications journal (2001)

17. Theussl, T., Möller, T., Gröller, M.E.: Optimal regular volume sampling. In: VIS '01: Proceedings of the conference on Visualization '01, Washington, DC, USA, IEEE Computer Society (2001) 91-98

18. Nagy, B.: Distance functions based on neighbourhood sequences. Publicationes Mathematicae Debrecen 63(3) (2003) 483-493

19. Strand, R., Nagy, B.: Some properties for distances based on neighbourhood sequences in the face-centered cubic grid and the body-centered cubic grid. Technical report, Centre for Image Analysis, Uppsala University, Uppsala, Sweden (2006) Internal Report 39. 